\title{
Herniación cerebral en oído medio
}

\section{Middle ear brain herniation}

\author{
José San Martín $\mathrm{P}^{1}$, Constanza Beltrán $\mathrm{R}^{1}$, Jorge Caro $\mathrm{L}^{1}$.
}

\begin{abstract}
RESUMEN
Se presenta un caso de herniación cerebral espontánea en oído medio, destacando la escasa e inespecífica sintomatología al momento de la consulta y su presentación bilateral. A continuación se presenta una breve revisión del tema haciendo mención a las etiologías, formas de presentación y tratamiento.

La herniación cerebral en oído medio es una patología poco común, siendo la etiología espontánea o idiopática, como es este caso, aun más infrecuente. La principal etiología es la posquirúrgica Si se sospecha su presencia, el paciente debe ser estudiado con tomografía computaday resonancia magnética de oídos. Se debe considerar estapatología en los casos que presenten ocupación atical o mastoídeajunto a una erosión del tegmen, sobre todo si el paciente ha sido operado previamente o tiene una otitis media crónica Una vez diagnostica da, se debe realizar una reparación quirúrgica precoz, debido al riesgo que se puedan generar complicaciones neurológicas. Para decidir el tipo de abordaje se deben considerar el tamaño de la herniación y del defecto, su localización y número. $\theta$ éxito de las distintas técnicas quirúrgicas es alto cuando se elige la técnica correcta.
\end{abstract}

Palabras clave: Hernia cerebral, tegmen timpani, resonancia magnética, cirugía.

\section{SUMMARY}

A case of spontaneous middle ear brain herniation is presented, enhancing the scarce and unspecified symptomatology at the time of consultation and its bilateral presentation. Below is presented a brief review of the matter mentioning the ethiologies, forms of presentation and treatment. Middle ear brain herniation is a fairly uncommon pathology, where spontaneous idiopatic ethiology, as is this case, is even more infrequent. The main ethiology is postsurgical. If its presence is suspected, the patient must be studied with computerized tomography and magnetic resonance of the ears. This pathology must be considered in the cases presenting atical or mastoid occupation together with tegmen erosion, specially if the patient has been previously operated or suffers from chronic middle ear otitis. Once diagnosed, an advanced surgical repair must be made due to the risk of generation of neurological complications. To decide on the type of approach, the size of the herniation and its defect, its location and number must be considered. Success with the different surgical techniques is high when the correct technique is selected.

Key words: Brain hernia, tegmen timpani, magnetic resonance, surgery.

\footnotetext{
${ }^{1}$ UDA Otorrinolaringología, Pontificia Universidad Católica de Chile.
} 


\section{CASO CLÍNICO}

Paciente de sexo masculino, de 52 años de edad, sin antecedentes mórbidos. Consultó inicialmente por cuadro consistente en otalgia izquierda de 2 días de evolución, asociado a fiebre hasta 38 grados e hipoacusia bilateral, además de un cuadro catarral con rinorrea serosa y decaimiento de 5 días de evolución. Al examen físico destacaba el tímpano izquierdo eritematoso y abombado, y el derecho opaco; el resto del examen ORL estaba dentro de límites normales. Se diagnosticó una otitis media aguda de oído izquierdo y una otitis media con efusión del oído derecho. En la Figura 1 se muestra la audiometría solicitada en esa consulta, donde se observa una hipoacusia de conducción bilateral. La impedanciometría mostró curvas "B" bilaterales.

Fue tratado con amoxicilina en dosis de $1 \mathrm{~g}$ cada 12 horas durante 10 días, con lo cual la otalgia y fiebre cedieron, aunque permaneció con hipoacusia A los 3 meses consultó nuevamente por persistir con hipoacusia bilateral. En esa oportunidad la otoscopía fue completamente normal.
Nuevas audiometría e impedanciometría mostraron persistencia de la hipoacusia de conducción y de las curvas "B", respectivamente.

Dada la falta de correlación entre el examen físico y los hallazgos audiométricos, se solicitó una tomografía computada de oídos (Figuras 2, 3, 4, 5).

La tomografía mostró ocupación de ambas cajas timpánicas, principalmente en la región del epitímpano, por un tejido con densidad de partes blandas, junto a un defecto de ambos tegmen timpani. La ocupación parecía ser continua con el tejido encefálico suprayacente. Se sospechó una hernia cerebral bilateral de oído medio y se solicitó una resonancia magnética de cerebro (Figuras 6, 7, 8).

La resonancia confirmó el diagnóstico al corroborar que la ocupación era continua e isodensa con el tejido cerebral.

\section{INTRODUCCIÓN}

La herniación de tejido cerebral dentro del hueso temporal fue documentada por primera vez en la literatura francesa por Caboche en 1902 ${ }^{1}$. En los

Figura 1.

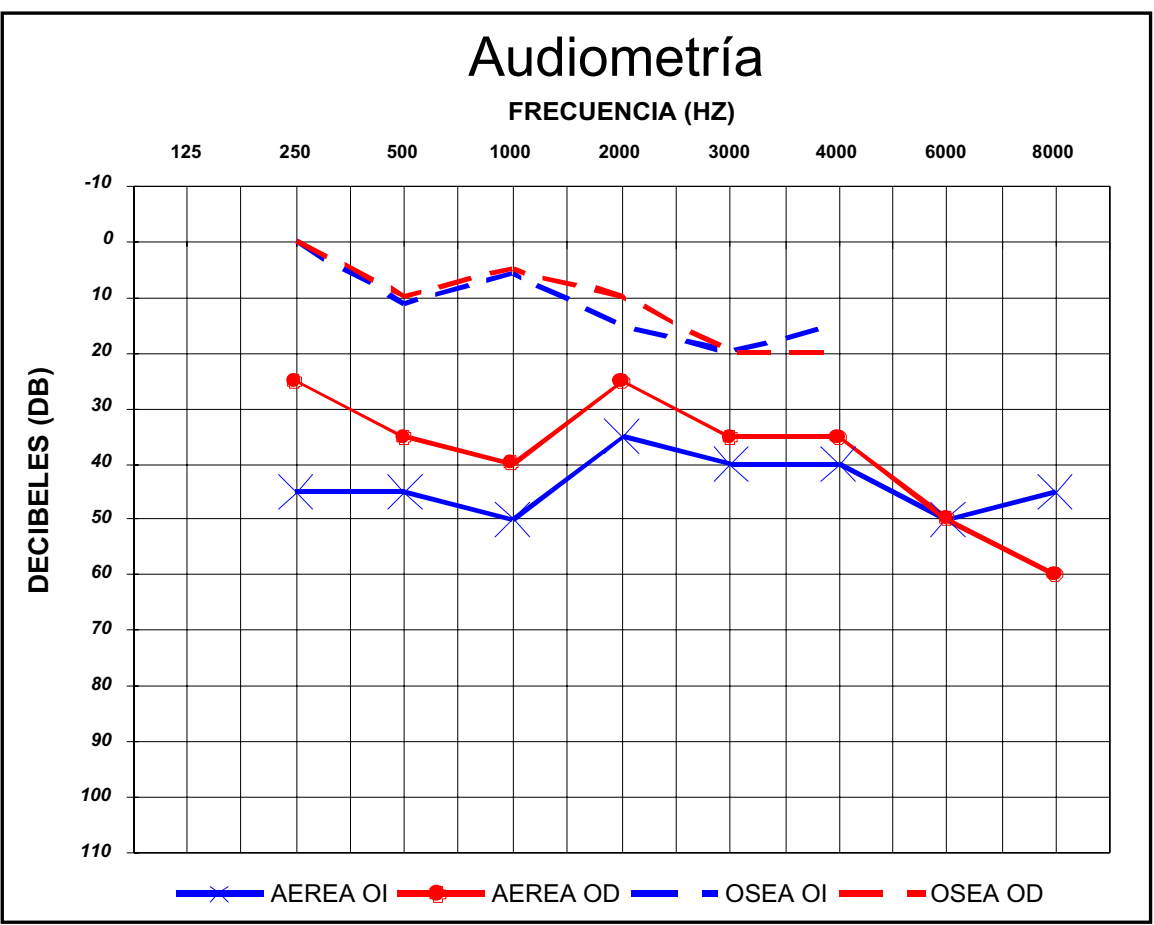




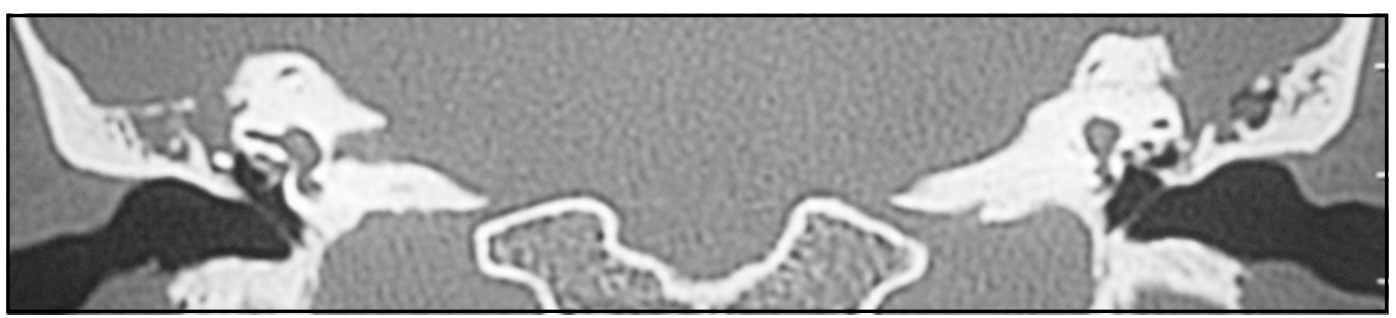

Figura 2.

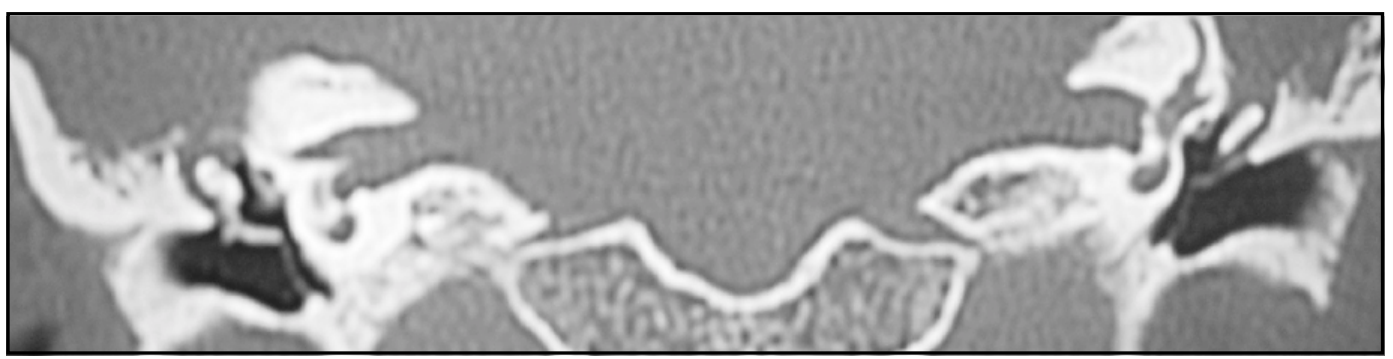

Figura 3.

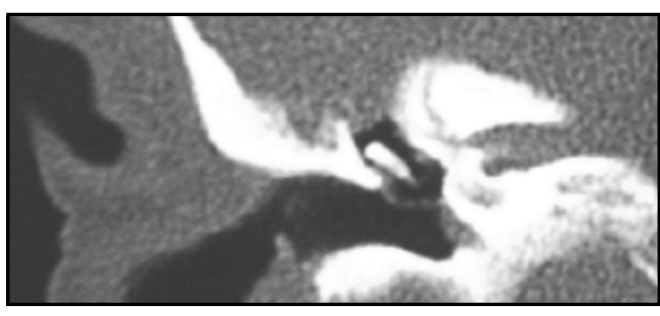

Figura 4.

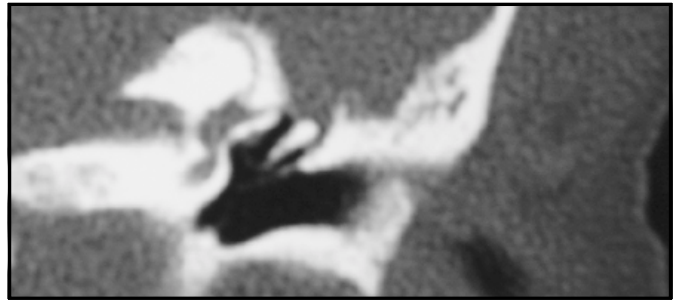

Figura 5.

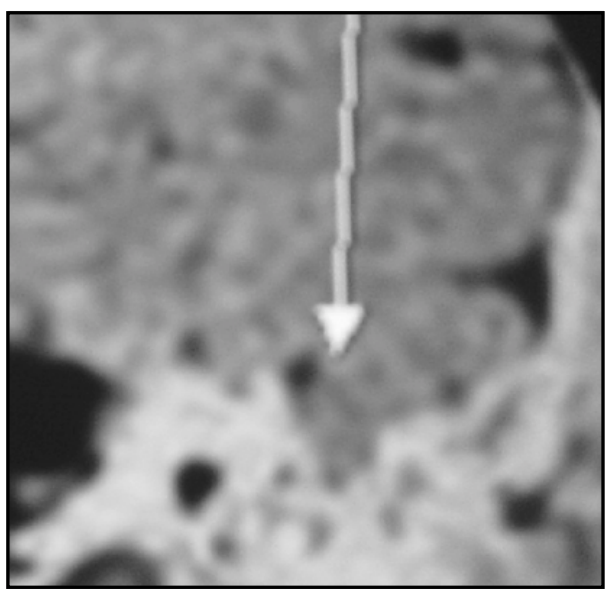

Figura 6. 


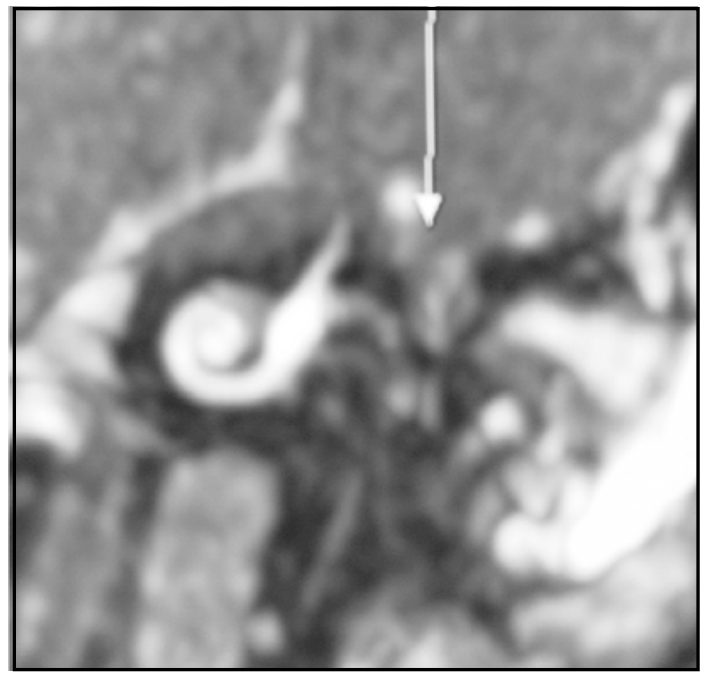

FGURA 7.

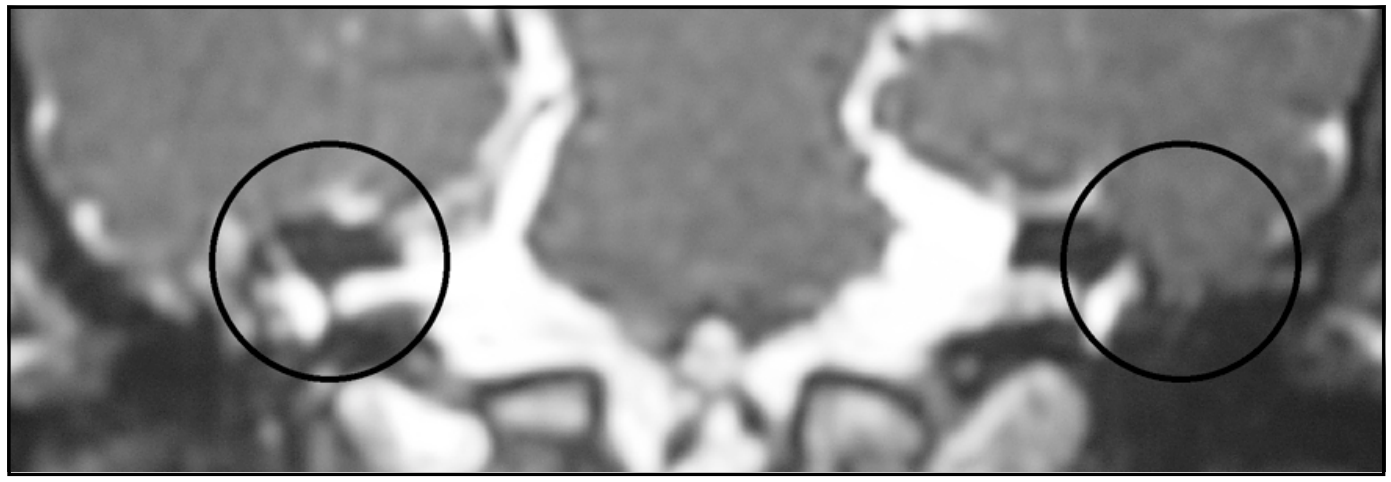

FGURA 8.

inicios del siglo $X X$, la presencia de una hernia cerebral en el oído medio se asociaba principalmente con el manejo quirúrgico de complicaciones intracraneanas de procesos infecciosos otogé nicos. Con la llegada de los antibióticos, la tomografía computada y los avances de la microcirugía, esta patología se ha convertido en una complicación rara de la cirugía de oído, pero aún está presente.

Según reportes en la literatura, el contenido herniario es meningoencefálico en el $74,1 \%$ de los casos, meníngeo en el 22,2\% y encéfalo aislado en el 3,7\% $\%^{2}$. La edad media de presentación es a los 38 años, con un rango que va desde los 6 a los 72 años; en el caso de la herniación espontánea (idiopática) la edad media de presentación es superior, situándose en torno a los 50 años. No parece haber diferencias según raza y es ligeramente más frecuente en mujeres que en hombres $(5: 4)^{2}$.

La principal etiología de la herniación cerebral en el oído medio es la posquirúrgica con un 
$58,97 \%$, le siguen la espontánea o idiopática con un $20 \%$, la postraumática con un $11,28 \%$, la postinfecciosa con un $9,23 \%$ y finalmente la secundaria a radionecrosis con un $0,51 \%{ }^{2}$.

\section{PATOGÉNESIS}

Un encefalocele congénito se produce por una alteración del proceso normal de osificación craneal, lo cual es también válido para los casos de encefaloceles espontáneos de oído. La incidencia reportada de dehiscencias óseas del tegmen llega al $34 \%$ en estudios de huesos temporales ${ }^{1}$. Sin embargo, el defecto óseo aislado no es suficiente para que se produzca la herniación, lo que se correlaciona con el hecho que la incidencia de hernias encefálicas en oído medio es mucho menor que la de dehiscencias del tegmen. Esto se debe a que también debe producirse una alteración a nivel de la duramadre. Se cree que variaciones normales de la presión intracraneana y largos períodos de exposición a pulsaciones repetidas en un mismo sitio podrían llevar finalmente al prolapso encefálico. Esto explicaría que una lesión aparentemente congénita aparezca en edades avanzadas de la vida.

También se ha propuesto la persistencia de tejido embrionario ectópico como origen de la herniación espontánea?.

En el caso del encefalocele secundario a una otitis media crónica, la reabsorción ósea y el compromiso dural se deberían a la acción del tejido de granulación y la inflamación crónica colesteatoma invade directamente el hueso y después de cierto tiempo, secundario a la acción enzimática prolongada, termina por debilitar también la duramadre ${ }^{1}$.

En el caso de la herniación traumática, o sea, la secundaria a traumatismos del hueso temporal con compromiso dural, podrían existir otros factores asociados como posibles infecciones, aumento transitorio de la presión intracraneana y alteración en la reparación del defecto óseo. $\mathrm{日}$ traumatismo de hueso temporal más frecuente- mente relacionado a la aparición de una hernia cerebral, es el iatrogénico asociado a la cirugía de mastoides ${ }^{1}$.

\section{DIAGNÓSTICO}

\section{Clínica}

La presentación clínica se relaciona en parte a la condición funcional del oído medio y la trompa de Eustaquio, pudiendo observarse efusión en oído medio, otorraquia o rinorraquia, siendo esta última la forma de más difícil diagnóstico ${ }^{3}$. La manifestación clínica más frecuente son los cuadros de otitis media con efusión a repetición y la hipoacusia de conducción $(\mathrm{HC})^{2}$. La HC puede ser no sólo secundaria a la efusión, sino también al efecto de masa de la hernia propiamente tal sobre la cadena osicular, alterando su motilidad ${ }^{3}$. 1 tinnitus también puede ser un síntoma de presentación, el que incluso puede ser pulsátil, debido al contacto de la masa con los huesecillos y a la transmisión del latido de la masa encefálica ${ }^{4}$. Oros síntomas menos frecuentes son vértigo, otalgia y plenitud aural ${ }^{1}$.

Ocasionalmente la herniación puede ser asintomática y descubrirse en una cirugía de revisión, principalmente de una mastoidectomía radical, siendo por lo tanto una forma de presentación de las hernias posquirúrgicas ${ }^{2}$. 日 $29,6 \%$ de las herniaciones meningoencefálicas en oído medio se diagnostican intraoperatoriamente ${ }^{3}$. $\mathrm{日}$ aspecto es el de una masa avascular dependiente del tegmen, de consistencia blanda, pulsátil, que se puede observar en caja timpánica, mastoides o conducto auditivo externo ${ }^{1,2}$.

La herniación también puede manifestarse a través de cuadros neurológicos, considerados muchas veces complicaciones, pero que ocasionalmente pueden ser la primera forma de presentación. Este es el caso de meningitis (que puede ser recurrente), convulsiones, paresia facial y afasia ${ }^{1-3}$ 


\section{Imágenes}

En caso de sospechar la existencia de una hernia cerebral en el oído medio, se debe solicitar una tomografía axial de oídos con cortes finos axiales y coronales, que permitirá el análisis de las estructuras óseas y la visualización del defecto del tegmen ${ }^{1-3}$, así como la presencia de un tejido con densidad de partes blandas en el oído medio. Frente a estos hallazgos, se debe hacer el diagnóstico diferencial entre herniación cerebral, colesteatoma, tejido de granulación, secreciones retenidas y granuloma de colesterol ${ }^{5}$. Sin embargo, la tomografía computada no permite hacer esta diferenciación, por lo que se debe complementar el estudio con una resonancia magnéti$\mathrm{ca}^{1-3,5}$.

En la resonancia magnética la herniación encefálica se verá isointensa con el parénquima cerebral, el meningocele se apreciará hipointenso en T1 e hiperintenso en T2, el colesteatoma aparecerá isointenso en T1 e hipointenso en T2 y el granuloma de colesterol será hiperintenso tanto en $\mathrm{T} 1$ como en $\mathrm{T}^{2,3}$. Sin embargo, el análisis aislado de las intensidades puede llevar a confusión en ciertos casos, ya que si el tejido encefálico herniado está edematoso o gliótico su intensidad puede ser similar a la de un colesteatoma; en estos casos el observar la continuidad del cerebro hacia el oído medio a través del defecto en el tegmen y la falta de cobertura meníngea son signos que orientan a la presencia de un encefalocele ${ }^{5}$.

\section{Otros estudios complementarios}

Frente a la sospecha de otorraquia o rinorraquia, el análisis del fluido permitirá decir si corresponde a líquido cefalorraquídeo (LCR). La cuantificación del contenido de glucosa puede ser útil. $\mathrm{日}$ LCR tiene normalmente una concentración de glucosa menor que la sérica pero mayor que la del moco nasal y las lágrimas. Una concentración de $30 \mathrm{mg} / \mathrm{ml}$ en el fluido (con glicemia simultánea normal) se considera positiva para LCR. Sin embargo, esta prueba tiene un alto índice de falsos positivos debido a la fácil contaminación del fluido con sangre o secreciones óticas o nasales 6 .

日 análisis del fluido en busca de la $B_{2}$ transferrina tiene alta sensibilidad y especificidad. La prueba consiste en realizar una electroforesis con inmunofijación para la transferrina, que detecta la isoforma de la proteína que es modificada por la neuraminidasa cerebral. La $B_{2}$ transferrina no se encuentra en otras secreciones ni en el suero ${ }^{2}$. Se requiere una cantidad muy pequeña de LCR para realizar la prueba; si la muestra está sin diluir basta con sólo $1 \mathrm{~mL}$ (1/50 de una gota) 6 .

\section{TRATAMIENTO}

Una vez diagnosticada una hernia cerebral en el oído medio se recomienda su corrección quirúrgica ${ }^{1,2}$.

Los objetivos de la cirugía incluyen: reestablecer la integridad de la bóveda intradural, evitar la fístula de LCR y evitar la recidiva'. Para cumplir con estos objetivos existen 3 tipos de abordajes: el transmastoídeo, mediante el cual se maneja la lesión desde "abajo", la vía de la fosa media, que maneja la lesión desde "arriba", y el abordaje combinado (trasmastoídeo más una minicraneotomía) $)^{1-3}$. La vía de la fosa media es la menos utilizada por implicar mayor riesgo quirúrgico y por no contar con la exposición mastoídea de la herniación. Las otras dos son las más aceptadas actualmente ${ }^{2,7}$.

Para decidir cuál es el mejor abordaje para cada caso se deben considerar factores como la extensión del defecto del tegmen y de la hernia, su localización y el número de defectos óseos ${ }^{1,6}$. Así, para hernias pequeñas y localizadas en mastoides, el abordaje transmastoídeo sería el indicado; por el contrario, si la herniación es de mayor tamaño y con un defecto amplio del tegmen $(>2 \mathrm{~cm})$, o se extiende hacia anterior acercándose al ápex petroso, o si se encuentran defectos múltiples en el tegmen, el abordaje combinado permitirá un mejor manejo de la patología ${ }^{1,6}$.

Respecto al manejo de la herniación propiamente tal, se debe considerar que en la mayoría de 
los casos corresponde a tejido cerebral desvitalizado y no funcionante ${ }^{1,3}$. 日 estudio histológico muestra un tejido con gliosis intensa, grados variables de degeneración y habitualmente inflamado'. Por otro lado, es frecuente que la hernia se encuentre en un ambiente infectado. Teniendo en cuenta estos hechos, la mayoría de los autores sugiere la coagulación bipolar del pedículo de la herniación y su posterior remoción ${ }^{1-3,7}$. En casos seleccionados de hernias con pedículos anchos, tejido cere bral de aspecto vital y sobre todo no infectado, se podría realizar su reducción $n^{3,7}$.

En cuanto a los materiales utilizados para la reparación del defecto, existen diversas posibilidades, pero se recomienda el uso de material autólogo, ya sea fascia temporal, hueso cortical, cartílago (del tabique nasal, por ejemplo) o músculo temporal ${ }^{1-3,7}$. Algunos autores complementan estos materiales con otros como cola de fibrina o Lyodura,,7.

En relación a la forma de reparar el defecto, la vía transmastoídea favorece la técnica extraduralintracraneal, para lo cual se debe levantar desde la mastoidectomía la duramadre adyacente al defecto óseo y luego interponer el material elegido (generalmente fascia temporal y/o cartílago), que debe ser de tamaño mayor al defecto ${ }^{7}$. Ouando en el abordaje combinado se utiliza una minicraneotomía, la reparación también es extraduralintracraneal, pero el injerto puede ser manipulado tanto intracranealmente como desde la mastoidectomía, por lo que esta técnica es mejor para defectos de mayor tamaño ${ }^{7}$. Si la craneotomía realizada es más amplia, algunos autores realizan una reparación intradural suturando el injerto de fascia temporal a la duramadre ${ }^{1,2}$; esto puede ser reforzado con otro injerto de fascia, cartílago o hueso colocado en situación extradural ${ }^{2}$. Los distintos injertos colocados pueden ser protegidos desde la mastoides mediante la rotación de un colgajo pediculado de músculo temporal ${ }^{2,7}$.

\section{CONCLUSIONES}

La herniación cerebral en el oído medio es una entidad rara, pero que el otorrinolaringólogo siempre debe considerar al recibir a un paciente con sintomatología ótica, sobre todo si éste tiene el antecedente de otitis media crónica o de haber sido sometido a una cirugía de oído. $日$ cirujano otólogo debe tenerla aún más presente si encuentra una masa en el conducto auditivo externo, caja timpánica o mastoides al realizar una cirugía de revisión, especialmente de una cirugía radical o timpanoplastía. Un exhaustivo análisis de las imágenes preoperatorias es imprescindible para un mejor enfrentamiento y planificación de la cirugía, la que debiera realizarse lo antes posible para evitar complicaciones. $\boxminus$ tipo de cirugía correctiva se decidirá según las características del defecto en el tegmen y de la herniación propiamente tal, prefiriendo un abordaje retromastoídeo para hernias únicas, pequeñas y de localización más posterior, y un abordaje combinado para hernias y defectos múltiples, de mayor tamaño o en zonas más anteriores del tegmen. Siguiendo estas recomendaciones se logrará el éxito quirúrgico.

\section{BIBLIOGRAFÍA}

1. JACKSON CG PAPPAS DG, ManOLIDIS $S$ et aL. Brain herniation into the middle ear and mastoid: concepts in diagnosis and surgical management. Am J Otol 1997; 18(2): 198-206.

2. AMORÓS SEAASTIÁ LI, BROTONS DURBÁN S, CARRASOO LLATAS M ET AL. Herniación meningoencefálica espontánea en oído medio: a propósito de dos casos. Acta Otorrinolaringol Esp 2002; 53: 507-12.

3. Vallicion JM, Grard N, Caces F, Bracoin F, MAGNAN J, GHAYS A. Idiopathic temporal encephalocele: report of two cases. Am J Otol 1999; 20(3): 390-3.

4. Kale SU, Pflederm AG, CradWick JC. Bilateral defects of the tegmen tympani associated with brain and dural prolapse in a patient with pulsatile tinnitus. J Laryngol Otol 2000; 114: 861-3.

5. KASET LG, SedenWurm DJ, Nimgding PH et AL. Magnetic resonance imaging of brain her- 
niation into the middle ear. Am J Otol 1992; 13(1): 74-7.

6. BROMN NE, GRUNDFAST KM, JABRE A, MEGRIIAN CA, OMALLEY BW, ROsenBrgG SI. Diagnosis and management of spontaneous cerebrospinal fluid middle ear effusion and otorrhea. Laryngoscope 2004; 114: 800-5.
7. F田STRA L, SANNA M, ZINI C, GamOlettI R, DeEOU P. Surgical treatment of brain herniation into the middle ear and mastoid. Am J Otol 1985; 6(4): 311-5. 